\title{
Piggy-back Hepatic Transplant Technique and Veno-venous Bypass Without Cardiac Arrest: A Multidisciplinary Approach in Borderline T3b/T3c Renal Tumors
}

\author{
Nechifor-Boila IA ${ }^{1 *}$, Suciu H², Loghin Andrada ${ }^{3}$, Borda Angela ${ }^{3}$, Maier A¹, Martha Orsolya ${ }^{1}$, Chibelean $\mathrm{C}^{1}$ \\ Department of Urology, Tîrgu-Mureș University of Medicine and Pharmacy, No.38 Gheorghe Marinescu Street, 540000, Tîrgu-Mures, Romania \\ 2 Department of Cardiovascular Surgery, Tîrgu-Mureș University of Medicine and Pharmacy, No.38 Gheorghe Marinescu Street, 540000 , Tîrgu-Mures, \\ Romania \\ 3 Department of Pathology, Tîrgu-Mureș University of Medicine and Pharmacy, No.38 Gheorghe Marinescu Street, 540000, Tîrgu-Mures, Romania
}

\begin{abstract}
Surgery for renal cell carcinomas with tumor thrombus extending in the Inferior Vena Cava (IVC) can be particularly challenging, especially in the retrohepatic and intraatrial situations (T3b and T3c). Classically, these tumors require the intraoperative use of cardio-pulmonary by-pass (CPB) and deep hypothermic circulatory arrest (DHCA), that can result in specific complications (stroke, platelet dysfunction), with increased postoperative morbidity rates.

In urological practice, a particular IVC preparation method is currently in use, allowing full control both upon the IVC and its tributaries. It is derived from the "piggy-back" liver transplantation technique and implies the resection of all hepatic ligaments, leaving the hepatic vascular connections intact. This procedure is joined by a form of veno-venous bypass (between the right atrium and the infrarenal IVC) that allows a constant central venous pressure (by assuring blood return), with less bleeding and without the need for CPB and DHCA (avoiding, in this way, their inherent complications). All in all, these recently-introduced procedures can offer better thrombus control, improved oncologic outcomes and smaller complication rates. We aim to present a case of borderline T3b/T3c renal tumor that was successfully treated in our university center using these techniques.
\end{abstract}

Keywords: liver transplant, tumor thrombus, renal cell carcinoma, veno-venous bypass

Received: 19 October 2014 / Accepted: 26 April 2015

\section{Introduction}

Renal Cell Carcinoma (RCC) is a highly vascular renal tumor with an unique tendency for vascular invasion and tumor thrombus formation, extending from the renal vein to the Inferior Vena Cava (IVC) and even the right atrium (1). It is estimated that 4 to $10 \%$ of RCCs have a tumor thrombus present in the venous system, while for $1 \%$ of the latter, the thrombus is extended to the right atrium $(1,2)$.

In terms of therapy, surgical resection remains the best option for this type of tumor, in spite of the great progress in oncologic treatment (immunotherapy, radiation and chemotherapy) (1). For RCCs with vascular invasion (T3a, $\mathrm{T} 3 \mathrm{~b}, \mathrm{~T} 3 \mathrm{c}$ ), the only potential cure in surgery, regardless of the level of the thrombus (I-IV), as long as there is no metastatic disease (the Neves staging system - see Table I). Thus, according to the 2014 edition of the European Association of Urology guidelines, the official recommendation is for radical nephrectomy and tumor thrombectomy for all T3 patients (1,3-6).

The surgical approach is variable according to each author but there is a consensus that such cases are complex,

* Correspondence to: Ioan Alin Nechifor-Boilă

E-mail: nechiforalin@yahoo.com require an excellent understanding of the local anatomy and benefit from an interdisciplinary approach $(1,7)$.

For borderline $\mathrm{T} 3 \mathrm{~b} / \mathrm{T} 3 \mathrm{c}$ renal tumors, classically, there is a need for cardio-pulmonary bypass and cardiac arrest in order to ensure a bloodless surgical field and optimum surgical comfort. However, this procedure implies a high risk of stroke and platelet dysfunction, resulting in the development of less-invasive bypass techniques together with more efficient approaches for renal resection (1,7-9).

Optimum caval exposure is a key element for an accurate renal resection which can be achieved by complete liver mobilization using a technique derived from the "piggyback" liver transplantation procedure, allowing thrombus management with only a veno-venous by-pass $(7,10-12)$.

The aim of our study was to present a case of borderline T3b/T3c renal tumor with a level III/IV thrombus (4) that was successfully treated in our university center using liver transplant techniques for caval exposure together with veno-venous by-pass.

\section{Case report}

We present the case of a 58 year-old female patient diagnosed with a right-sided, borderline $\mathrm{T} 3 \mathrm{~b} / \mathrm{T} 3 \mathrm{c}$ renal cell carcinoma in our department. At presentation, the patient complained of weight loss and fatigability. The physical 
Table I. The Neves clinical staging system for renal tumors with tumor thrombi involving the renal vascular system and the IVC, in comparison to the current TNM classification issued by the Union for International Cancer Control in 2009.

\begin{tabular}{cll}
\hline $\begin{array}{c}\text { Thrombus level (Neves) } \\
{[4,15]}\end{array}$ & TNM [6] & Description \\
\hline 0 & T3A & The thrombus is in the renal vein \\
I & T3B & The thrombus invades the IVC, extending $<2 \mathrm{~cm}$ above renal vein \\
II & The thrombus invades the IVC, extending $>2 \mathrm{~cm}$ above renal vein but below the hepatic veins \\
III & The thrombus invades the IVC, extends above the hepatic veins but below the diaphragm \\
IV & T3C & The thrombus invades the IVC, extends above the diaphragm \\
\hline
\end{tabular}

examination found no abnormalities in the abdomen. An abdominal ultrasound examination was performed and revealed a locally advanced, right-sided renal tumor. A high resolution angio-CT scan highlighted a tumor thrombus advancing into the inferior vena cava to the level of the diaphragm (Figure $1 \mathrm{~A}$ and $\mathrm{B}$ ). No signs of IVC wall invasion from the thrombus nor metastatic disease were found.

Surgical treatment was initiated and a combined urological-cardiovascular surgical team was formed. A modified chevron incision was used, later completed with a midline sternotomy.

The parietal peritoneum, lateral to the ascendant colon, was incised and the right colon and mesocolon were deflected. Next, Gerota's fascia was exposed and incised, revealing the perirenal space. The right kidney was gradually mobilized after having ligated the large array of small collateral veins in the perirenal atmosphere. The right ureter and the right ovarian vein were divided. The same was per-
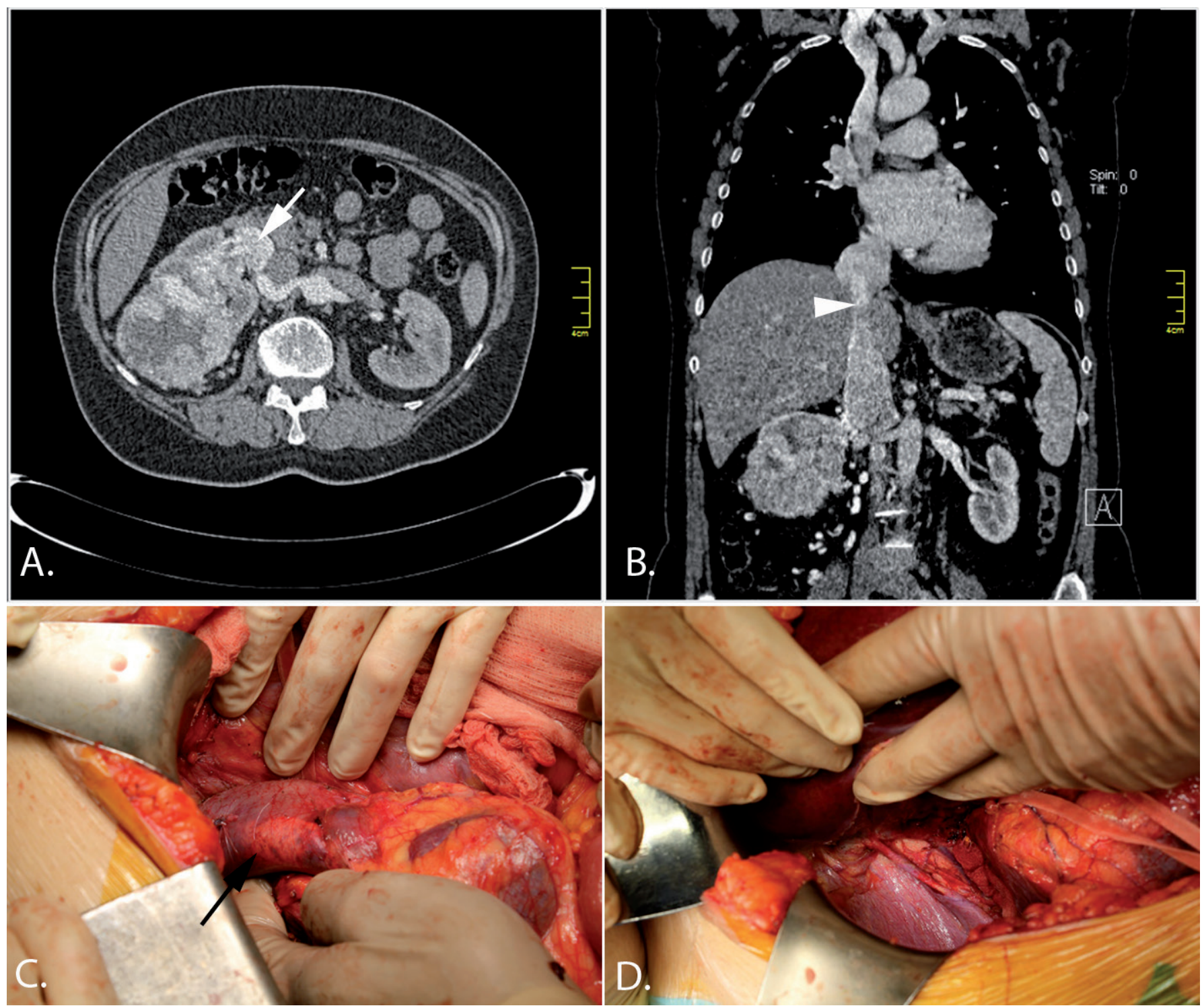

Fig. 1. Contrast-enhanced CT examination of the patient showing the right-sided renal tumor with the enhancing thrombus in the arterial phase (1 A - white arrow). The coronal reconstruction from the same examination in the venous phase highlights the thrombus extending to diaphragm (1B-arrowhead). After a thorough exposure of the IVC, the thrombus can be observed by the transparency of the caval wall (1C - black arrow). Liver transplant techniques were used and the liver was deflected leftwards, exposing the entire IVC (1D). 
formed for the right renal artery. Next, the subhepatic IVC was exposed by removing all perivascular fatty tissues. The lumbar vessels were identified, clamped and resected. After clearing the subhepatic IVC, the tumor thrombus was identified extending to the retrohepatic space (Figure 1C).

The liver was mobilized according to the actual "piggyback" liver transplantation technique in order to obtain complete exposure of the IVC. The teres, right coronal, falciform and triangular ligaments were divided and the right hepatic lobe was deflected leftwards (figure 1D). The posterior parietal peritoneum was incised and full access to the IVC and suprahepatic veins was obtained. By palpation, the tumor thrombus was identified reaching over the caval hiatus of the diaphragm. The decision for venovenous by-pass was taken.

The cardiovascular team performed sternotomy and exposed the anterior mediastinum. The pericardium was incised, exposing the right atrium and terminal IVC. The right atrium and the infrarenal IVC were cannulated. Pringle's maneuver was undertaken. After two minutes (in order to allow hepatic decompression), the infrarenal IVC, the left renal vein, suprahepatic veins and intrapericardic IVC were consecutively occluded, ensuring a "bloodless IVC". By-pass was initiated (Figure 2A). The thrombus was "milked" downwards to a level inferior to the suprahepatic veins. The intrapericardic IVC clamp was released and repositioned below the suprahepatic veins. A circumferential cavotomy was performed around the right renal
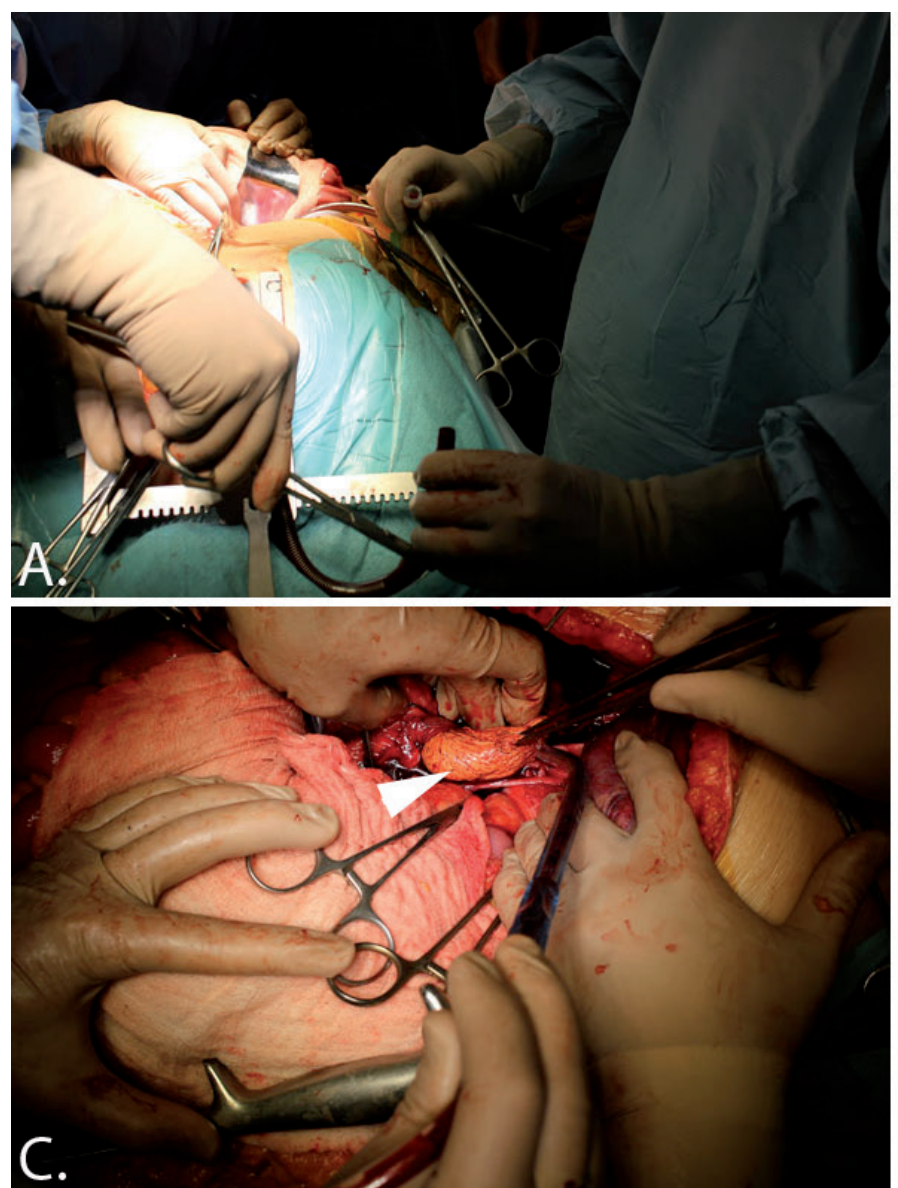

Fig. 2. After canullation of both infrarenal IVC and the right atrium, the veno-venous bypass is prepared (2A). After cavotomy, the thrombus is exposed (2B-arrowhead) and extracted (2C-arrowhead) together with the right kidney. Its intergrity is checked before suturing the IVC (2D).

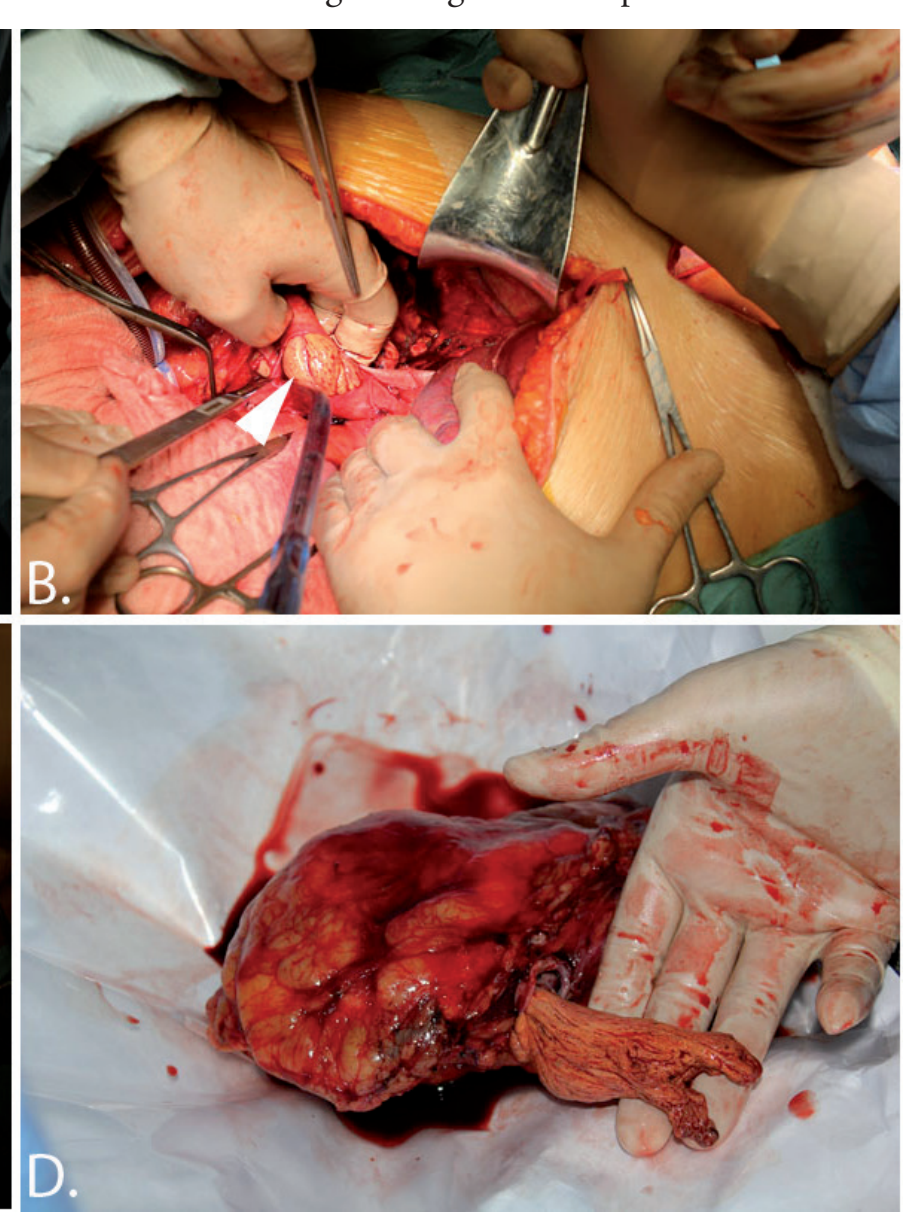

vein running upwards to the infrahepatic clamp. The right kidney together with the thrombus were resected en-bloc (Figure $2 \mathrm{~B}$ and 2C). The IVC was flushed and thrombus checked for integrity (Figure 2D). After assurance of complete removal, the IVC was closed using a running 4.0 polypropylene suture. All clamps on the IVC and its branches were removed. By-pass was suppressed. Drains were put into position and both abdominal and thoracic incisions were closed. Blood loss was kept to a minimum. The patient had a favorable outcome and was discharged from hospital 12 days later. At subsequent follow-up visits, the patient was cancer free at 3, 6 and 12 months.

\section{Discussion}

We presented a case of borderline $\mathrm{T} 3 \mathrm{~b} / \mathrm{T} 3 \mathrm{c}$ renal tumor, with a type III/IV thrombus that was successfully treated using liver transplantation methods for optimum IVC exposure. By using a veno-venous by-pass, deep hypothermic circulatory arrest and cardio-pulmonary bypass were not necessary, thus avoiding potentially severe complications.

Surgical management of a RCC with thrombus extension to the retrohepatic vena cava is challenging. For oncologic purposes, radical nephrectomy and tumor thrombectomy must be performed with clear surgical margins while assuring the safety of the patient (7).

Thrombus management is the most difficult part of the operation, but it is critical for favorable outcomes. Embolism and hemorrhage during IVC manipulation are serious 
complications that can have even life-threatening consequences, implying increased morbidity rates, transfusions and even further procedures (7).

Our technique is similar to the one proposed by Ciancio et al, with a difference residing in our need for veno-venous bypass. Their team propose a resection procedure that is purely abdominal, with a similar IVC exposure technique but without bypass (7).

The same technique was also reported by Gorin et al who obtained very good results in the treatment of RCCs presenting with level II thrombi (13).

Wotkowicz et al, in their broad review on renal tumors invading the vena cava presented their broad experience with both classical and minimally invasive by-pass, with recognition of the potential bypass-related complications like coagulopathy and stroke. When comparing the two by-pass methods, the team reported shorter operative and hospital times, decreased transfusion rates and less mechanical ventilatory support in favor of the minimallyinvasive one $(1,14)$.

\section{Conclusion}

The use of liver transplantation techniques for radical nephrectomy in borderline $\mathrm{T} 3 \mathrm{~b} / \mathrm{T} 3 \mathrm{c}$ renal tumors, with or without the use of veno-venous by-pass, is an efficient technique, allowing improved vascular control, less risk of embolism, adequate venous return and a decreased blood loss.

\section{Conflicts of interest statement}

There are no conflicts of interest.

\section{Acknowledgements}

Written informed consent was obtained from the patient to publish both in print and online.

This paper is supported by the Sectoral Operational Programme Human Resources Development (SOP HRD), financed from the European Social Fund and by the Romanian Government under the contract number POSDRU/159/1.5/S/133377/

\section{References}

1. Wotkowicz C, Wszolek MF, Libertino JA. Resection of renal tumors invading the vena cava. Urol Clin North Am, 2008;35:657-671.

2. Kim HL, Zisman A, Han KR, Figlin RA, Belldegrun AS. Prognostic significance of venous thrombus in renal cell carcinoma. Are renal vein and inferior vena cava involvement different? J Urol, 2004; 171:588-591.

3. Whitson JM, Reese AC, Meng MV. Population based analysis of survival in patients with renal cell carcinoma and venous tumor thrombus. Urol Oncol, 2013;31:259-263.

4. Neves RJ, Zincke H. Surgical treatment of renal cancer with vena cava extension. Br J Urol, 1987;59:390-395.

5. Moinzadeh A, Libertino JA. Prognostic significance of tumor thrombus level in patients with renal cell carcinoma and venous tumor thrombus extension. Is all T3b the same? J Urol, 2004;171:598-601.

6. Ljungberg B, Bensalah K, Bex A. Guidelines on Renal Cell Carcinoma, European Association of Urology, Available from: http://www.uroweb.org/ gls/pdf/10\%20Renal\%20Cell\%20Carcinoma_LR.pdf

7. Ciancio G, Gonzalez J, Shirodkar SP, Angulo JC, Soloway MS. Liver transplantation techniques for the surgical management of renal cell carcinoma with tumor thrombus in the inferior vena cava: step-by-step description. Eur Urol, 2011;59:401-406.

8. Ciancio G, Shirodkar SP, Soloway MS, et al. Renal carcinoma with supradiaphragmatic tumor thrombus: avoiding sternotomy and cardiopulmonary bypass. Ann Thorac Surg, 2010;89:505-510.

9. Gorin MA, Gonzalez J, Garcia-Roig M, Ciancio G - Transplantation techniques for the resection of renal cell carcinoma with tumor thrombus: A technical description and review. Urol Oncol, 2013;31:1780-1787.

10. Botea F, Brasoveanu V, Constantinescu Aet al. - Living donor liver transplantation with dual grafts -- a case report. Chirurgia (Bucur), 2013;108:547-552.

11. Borz C, Marian D, Bara TJret al. - Anatomical and Surgical Basis for Adult Living Donor Liver Transplantation with the Right Liver Lobe. Acta Medica Marisiensis, 2014;60:129-132.

12. Popescu I, Ionescu M, Tulbure D, Ciurea S, Băilă S, Brașoveanu V, Hrehoreț D, Sârbu-Boeți P, Pietrăreanu D, Alexandrescu S, Dorobanțu B, Gheorghe L, Gheorghe C, Mihăilă M, Boroș M, Croitoru M, Herlea V. Orthotopic liver transplantation in adult patients with cadaveric grafts. Experience of the Fundeni Center of General Surgery and Liver Transplantation. Chirurgia (Bucur), 2005;100:13-26.

13. Gorin MA, Garcia-Roig M, Shirodkar SP, Gonzalez J, Ciancio G - Modified surgical technique for the management of renal cell carcinoma with level I or II tumor thrombus. Urology, 2012;79:478-481.

14. Wotkowicz C, Libertino JA, Sorcini A, Mourtzinos A - Management of renal cell carcinoma with vena cava and atrial thrombus: minimal access vs median sternotomy with circulatory arrest. BJU Int, 2006;98:289-297.

15. Pouliot F, Shuch B, Larochelle JC, Pantuck A, Belldegrun AS. Contemporary management of renal tumors with venous tumor thrombus. J Urol, 2010;184:833-841. 